\title{
Transient MHD Radiative Fluid Flow over an Inclined Porous Plate with Thermal and Mass Diffusion: An EFDM Numerical Approach
}

\author{
Noor Alam ${ }^{1}$, Saykat Poddar ${ }^{2}$, M Enamul Karim ${ }^{3}$, Mohammad Sanjeed Hasan², Giulio Lorenzini ${ }^{*}$ \\ ${ }^{1}$ Department of Mathematics, Faculty of Basic Science, Primeasia University, Dhaka 1213, Bangladesh \\ ${ }^{2}$ Department of Mathematics, Faculty of Science, Bangabandhu Sheikh Mujibur Rahman Science and Technology University, \\ Gopalganj 8100, Bangladesh \\ ${ }^{3}$ Department of Mathematics, Comilla University, Cumilla 3506, Bangladesh \\ ${ }^{4}$ Department of Engineering and Architecture, University of Parma, Parco Area delle Scienze 181/A, Parma 43124, Italy
}

Corresponding Author Email: giulio.lorenzini@unipr.it

https://doi.org/10.18280/mmep.080508

Received: 3 July 2021

Accepted: 27 September 2021

\section{Keywords:}

MHD, inclined porous plate, heat and mass

diffusion, Soret effect, Dufour effect

\begin{abstract}
This research aims to delve into the transient MHD flow over a porous plate having an inclination, with heat and mass diffusion by taking the radiative phenomenon into consideration. The flow controlling equations of continuity, momentum, energy, and concentration are developed using the boundary layer approximations. The radiative flux is described using a differential approximation. The governing time-dependent equations are brought into a conversion to create a system of non-dimensional partial differential equations (PDEs). Numerical schemes approaching the explicit finite difference method (EFDM) are employed to discretize and reckon the equations in dimensional agreement. Stability and convergence checking are prepared to ensure the converging restrictions of pertinent parameters. The profiles of velocity, concentration, and temperature have been illustrated graphically and discussed comprehensively.
\end{abstract}

\section{INTRODUCTION}

Investigating the magnetohydrodynamic flow of an electrical conduction fluid on a highly heated surface has sparked many investigators' interest in some in-depth implementations in the fields of engineering, for instance, the petroleum industry and reactors. The foremost official use of the term magnetohydrodynamics has come from Alfvén [1]. Sheri and Modugula [2] explored mass and energy diffusion on transient MHD flow where the chemical reaction in the fluid has been taken into account. This finite element approach reported a salient impact of the Dufour-Soret pair on the characteristics of flow and transport. Makinde et al. [3] demonstrated and reckoned the EFDM result of an electrically and thermally responsive and viscous fluid where fluid passes through two parallel porous plates by maintaining a convective energy exchange within the surroundings. Subhas Abel and Begum [4] presented the large Prandtl number's case on the flow of viscoelastic materials through a sheet of stretching property. The heat transfer criteria have been analytically counted by the conversion of the energy equation into the version of a hypergeometric differential equation. Chamkha et al. [5] considered tri-diagonal-based numerical schemes to analyze the steady-state situation of MHD flow containing non-isothermal property in the existence of radiation phenomenon. Raptis [6] investigated the vertically constrained flow under the action of relatively higher temperature and thermal radiation. Ahmed and Iqbal [7] employed an annular duct to investigate the power-law nanofluid flow for immutable pressure gradient. They have found a relatively higher hiking trend in the change of heat transfer rate for the shear thinning case. Kiyasatfar and Pourmahmoud [8] iteratively determined non-Newtonian flow within squareshaped microchannels by emphasizing the dissipation and joule heating phenomena. Ravikumar et al. [9] inspected the time-independent Couette flow with the suction velocity varying with periodicity by incorporating the perturbation technique.

Flow within a medium of porosity due to the shifting of a vertical wall with radiation has been researched by Raju et al. [10]. The existence of a permeability medium was found to be tended the fluid velocity up. Later, Reddy et al. [11] researched the suction case of MHD chemically responsive and radiative flow by taking the Laplace transformation technique as the solution method. Reddy et al. [12] took an exponentially moving vertical plate to analyze the transient MHD reactive flow under the workings of radiation absorption. Izadi et al. [13] executed a rigorous numerical inspection on nanofluid flow involving microorganisms wielding through a stretching surface. The Lewis number has been found to lead the transfer of microorganisms afar from the surface of the sheet. Islam et al. [14] executed numerical counting on the MHD densitydependent flow with the magnetic field induction. Mollah et al. [15, 16] have researched the MHD in-between flow of Bingham materials through parallel Riga and/or porous plates. Omeshwar Reddy et al. [17] examined the features of thermal diffusion, diffusion-thermo, and radiative behavior on flow with MHD fact. Pandya and Shukla [18] worked on timedependent MHD flow through a permeable medium to exhibit the mixed impacts of thermal diffusion, diffusion-thermo and radiation phenomena. Omokhuale et al. [19] researched a convective and transient MHD Couette flow and draw a numerical description of the flow properties by using the finite element numerical procedure. Magnetohydrodynamics 
stagnation point flow of chemically reactive fluid maintaining Casson rheology for nonlinear movement of a sheet has been modeled by Khan et al. [20]. They reported an adverse correlation between velocity profile and magnetic parameter. Waqas et al. [21] analyzed how micropolar liquid flows towards a nonlinear stretched surface with the help of convective boundary conditions. Malga and Kishan [22] researched polar incompressible flow through a permeable field of porosity, directed in the longitudinal accession for time-alterable suction velocity. With the involvement of thermal radiation and heat generation impacts along with MHD criteria, Makinde [23] overviewed the stagnation point flow. Anand et al. [24] researched Couette flow through two parallel silver metals with uniform and periodic suction at the lower one. This stream function-based analytical study reported that the axial flow velocity increase by the strengthening of the Hartmann number. Tassaddiq [25] discussed MHD fluid movement over a heated surface by forming fractional PDEs and solved it through the algorithm of Zakian's and Laplace's numerical approach. The fractional criteria were mentioned to suppress the velocity and also mooted that the minimal velocity occurs when the fractional parameter is exactly one. The destructive chemically reactive flow of transient MHD criteria dipped in the permeable field for the movement of an inclined plate has been studied by Pattnaik et al. [26]. Retardation of velocity was reported to be sustained for the inclination angle and heat generation while the permeable field worked to create a barrier against the backflow. Sabu et al. [27] reviewed the Hall current operation on MHD Ferro-nanofluid by means of a channel flow of inclination where the oscillating nature of boundary layers has been involved. The skin friction, in that study, was marked to have a negative influence due to the Hall impact as well as the existence of a heat source. Khashi'ie et al. [28] researched the flow of $\mathrm{Cu}-\mathrm{Al}_{2} \mathrm{O}_{2}-\mathrm{H}_{2} \mathrm{O}$ nanofluid with Joule heating criteria. The stability of the flow leads the study to choose the reliable solution among the possible two solutions and strengthening of suction intensify to the heat transfer.

Poddar et al. [29] investigated the suction case of MHD vertically constrained flow with magnetic field induction and responsive radiation as well as dissipating features. Both the thermal diffusion and diffusion-thermo impacts have been reported to point significant changes in fluid concentration. Ouaf [30] examined thermal radiation on the MHD steady flow which is asymmetric. Thermal radiation on flow nature has been mentioned on sundry studies as an energy-boosting fact and some studies are worth mentioning, as suggested in Refs. [31-34]. Further, the EFDM schemes have been taken by many researchers [35-38] to analyze multifarious geometries of flow under different circumstances.

This study motives to explore the transient MHD flow over a plate of inclination having porosity, with thermal and mass diffusion characteristics. The interaction of the imposed magnetic field and fluid conductivity induce a magnetic field which has been kept out from the current study for the insignificant estimation of magnetic Reynolds number. Here, the numerical results of the EFDM schemes have been calculated with the mixed impacts of thermal and mass diffusion in two-dimensional transient flow of an incompressible fluid. The behavior of flow properties for several pertinent parameters have been discussed graphically. Finally, a conclusion has been drawn focusing on the main theme of the current volume.

\section{MATHEMATICAL MODEL OF THE FLOW}

Consider, a transient MHD laminar flow with the functionality of Dufour and Soret influences and the fluid is viscous and incompressible in nature. In Figure $1, X$ axis represents the direction of fluid motion and this axis is measured along the porous plate. An inclination $\alpha$ is considered between $X$ and $Y$ directions. The temperature and concentration variable are $T_{w}$ and $C_{w}$ respectively at the wall of the plate occupied with radiative fluid of uniform ambient temperature $T_{\infty}$ and uniform ambient concentration $C_{\infty}$

The uniform magnetic field is denoted by $\mathbf{B}$ that is imposed to $y=0$ along the $Y$ axis and deemed to be electrically nonconducting. Let, the magnetic vector field is, $\mathbf{B}=$ $\left(B_{x}, B_{y}, B_{z}\right)=\left(0, B_{0}, 0\right)$ where the components are $B_{x}, B_{y}, B_{z}$ in the $X, Y, Z$-direction, respectively.

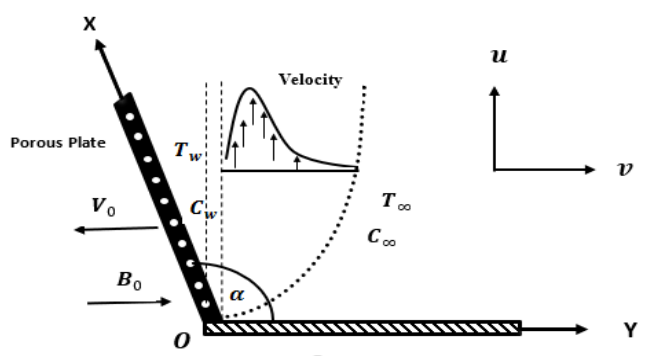

Figure 1. Configuration of the flow model

The governing equations for the two-dimensional transient flow problem are:

Continuity equation:

$$
\frac{\partial u}{\partial x}+\frac{\partial u}{\partial y}=0
$$

Momentum equation:

$$
\begin{aligned}
& \frac{\partial u}{\partial t}+u \frac{\partial u}{\partial x}+v \frac{\partial u}{\partial y}=\vartheta \frac{\partial^{2} u}{\partial y^{2}}-\frac{1}{\rho} \sigma B_{0}{ }^{2} u+g \beta\left(T-T_{\infty}\right) \\
& \cos \alpha+g \beta^{*}\left(C-C_{\infty}\right) \cos \alpha
\end{aligned}
$$

Energy equation:

$$
\begin{aligned}
& \frac{\partial T}{\partial t}+u \frac{\partial T}{\partial x}+v \frac{\partial T}{\partial y}=\frac{k}{\rho c_{p}} \frac{\partial^{2} T}{\partial y^{2}}+\frac{1}{\rho c_{p}} \sigma B_{0}{ }^{2} u^{2} \\
& +D_{m} \frac{\partial^{2} C}{\partial y^{2}}-\frac{1}{\rho c_{p}} \frac{\partial q_{r}}{\partial y}
\end{aligned}
$$

Concentration equation:

$$
\frac{\partial C}{\partial t}+u \frac{\partial C}{\partial x}+v \frac{\partial C}{\partial y}=D_{m} \frac{\partial^{2} C}{\partial y^{2}}+D_{T} \frac{\partial^{2} T}{\partial y^{2}}
$$

where, $D_{m}$ is the mass diffusivity, and $q_{r}$ is the radiative heat flux, $\vartheta$ is the kinematic viscosity, $\sigma$ is the electric conductivity, $\frac{k}{\rho c_{p}}$ is the thermal diffusivity, $\rho$ is the fluid density, $k$ is the thermal conductivity, and $c_{p}$ is the specific heat at constant pressure.

The boundary conditions are subjected as, 


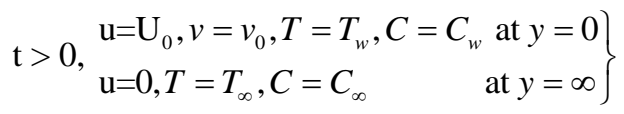

Rosseland approximation of radiative flux generates [29],

$$
q_{r}=-\frac{4 \sigma_{1}}{3 k_{1}} \frac{\partial T^{4}}{\partial y}
$$

where, $\sigma_{1}$ is the Stefan-Boltzmann constant. Expansion of $T^{4}$ in terms of $T_{\infty}$ by taking the Taylor series and avoiding the higher-orders, we have [38],

$$
T^{4} \approx 4 T_{\infty}^{3} T-3 T_{\infty}^{4}
$$

The Eq. (3) has been refurnished as:

$$
\left.\begin{array}{l}
\frac{\partial T}{\partial t}+u \frac{\partial T}{\partial x}+v \frac{\partial T}{\partial y}=\frac{k}{\rho c_{p}} \frac{\partial^{2} T}{\partial y^{2}}+\frac{1}{\rho c_{p}} \sigma B_{0}{ }^{2} u^{2} \\
+D_{m} \frac{\partial^{2} C}{\partial y^{2}}+\frac{16 \sigma_{1} T_{\infty}^{3}}{3 \rho c_{p} k_{1}} \frac{\partial^{2} T}{\partial y^{2}}
\end{array}\right\}
$$

\section{MATHEMATICAL FORMULATION}

To discretized and reckon the flow governing equations by using the EFDM procedure, it is necessary to convert them into dimensionless form.

Now we take some dimensionless variables:

$$
\begin{aligned}
& X=\frac{x U_{0}}{\vartheta}, Y=\frac{y U_{0}}{\vartheta}, U=\frac{u}{U_{0}}, V=\frac{v}{U_{0}}, \tau=\frac{t U_{0}^{2}}{\vartheta}, \\
& \theta=\frac{T-T_{\infty}}{T_{w}-T_{\infty}}, \varphi=\frac{C-C_{\infty}}{C_{w}-C_{\infty}}
\end{aligned}
$$

The governing Eqns. (1), (2), (8), and (4) are transformed as follows:

$$
\begin{gathered}
\frac{\partial U}{\partial X}+\frac{\partial V}{\partial Y}=0 \\
\frac{\partial U}{\partial \tau}+U \frac{\partial U}{\partial X}+V \frac{\partial U}{\partial Y}=\frac{\partial^{2} U}{\partial Y^{2}}-M U \\
+G_{r} \theta \cos \alpha+G_{m} \varphi \cos \alpha \\
\frac{\partial \theta}{\partial \tau}+U \frac{\partial \theta}{\partial X}+V \frac{\partial \theta}{\partial Y}=\frac{1+N_{r}}{P_{r}} \frac{\partial^{2} \theta}{\partial Y^{2}}+M E_{c} U^{2}+D_{u} \frac{\partial^{2} \varphi}{\partial Y^{2}} \\
\frac{\partial \varphi}{\partial \tau}+U \frac{\partial \varphi}{\partial X}+V \frac{\partial \varphi}{\partial Y}=\frac{1}{S_{c}} \frac{\partial^{2} \varphi}{\partial Y^{2}}+S_{r} \frac{\partial^{2} \theta}{\partial Y^{2}}
\end{gathered}
$$

And boundary conditions:

$$
\left.\begin{array}{l}
\tau>0, U=1, V=P, \theta=1, \varphi=1 \text { at } Y=0 \\
U \rightarrow 0, V \rightarrow 0, \theta \rightarrow 0, \varphi \rightarrow 0 \text { at } Y \rightarrow \infty .
\end{array}\right\}
$$

where,

$M=\frac{1}{\rho} \frac{\sigma B_{0}^{2} \vartheta}{U_{0}^{2}}$ is the magnetic Parameter;

$G_{r}=\frac{\vartheta g \beta\left(T_{w}-T_{\infty}\right)}{U_{0}{ }^{3}}$ is the Grashof number of heat transfer;

$G_{m}=\frac{\vartheta g \beta^{*}\left(C_{w}-C_{\infty}\right)}{U_{0}{ }^{3}}$ is the Grashof number of mass transfer;

$N_{r}=\frac{16 \sigma_{1} T_{\infty}^{3}}{3 k_{1} k}$ is the radiation parameter;

$P_{r}=\frac{\rho C_{p} \vartheta}{k}$ is the Prandtl number;

$E_{c}=\frac{U_{0}{ }^{2}}{C_{p}\left(T_{w}-T_{\infty}\right)}$ is the Eckert number;

$D_{u}=\frac{D_{m}\left(C_{w}-C_{\infty}\right)}{\vartheta\left(T_{w}-T_{\infty}\right)}$ is the Dufour number;

$S_{c}=\frac{\vartheta}{D_{m}}$ is the Schmidt number;

$S_{r}=\frac{D_{T}\left(T_{w}-T_{\infty}\right)}{\vartheta\left(C_{w}-C_{\infty}\right)}$ is the Soret number;

$P=\frac{v_{0}}{U_{0}}$ is the suction parameter.

\section{STABILITY AND CONVERGENCE}

In this part, we discuss the stability and convergence analysis. Here the continuity equation is not considered as $\Delta \tau$ does not seem. Fourier expansion for $U, \theta$ and $\varphi$ at $t=0$ are:

$$
\left.\begin{array}{l}
U: \Psi(\tau) e^{i \alpha X} e^{i \beta Y} \\
\theta: \xi(\tau) e^{i \alpha X} e^{i \beta Y} \\
\varphi: \eta(\tau) e^{i \alpha X} e^{i \beta Y}
\end{array}\right\}
$$

And after the time-step,

$$
\left.\begin{array}{l}
U: \Psi^{\prime}(\tau) e^{i \alpha X} e^{i \beta Y} \\
\theta: \xi^{\prime}(\tau) e^{i \alpha X} e^{i \beta Y} \\
\varphi: \eta^{\prime}(\tau) e^{i \alpha X} e^{i \beta Y}
\end{array}\right\}
$$

Now substituting all the above Eqns. (14) and (15) into the Eqns. (10)-(12). Further, upon simplification, the governing equations have been obtained as:

$$
\left.\begin{array}{l}
\frac{\Psi^{\prime}(\tau)-\Psi(\tau)}{\Delta \tau}+U \frac{\Psi(\tau)\left(1-e^{-i \alpha \Delta X}\right)}{\Delta X}+V \frac{\Psi(\tau)\left(e^{i \beta \Delta Y}-1\right)}{\Delta Y}= \\
G_{r} \xi^{\prime}(\tau) \cos \alpha+G_{m} \eta^{\prime}(\tau) \cos \alpha-M \Psi(\tau)+\frac{2 \Psi(\tau)(\cos \beta \Delta Y-1)}{(\Delta Y)^{2}}
\end{array}\right\}
$$$$
\left.\begin{array}{c}
\frac{\xi^{\prime}(\tau)-\xi(\tau)}{\Delta \tau}+U \frac{\xi(\tau)\left(1-e^{-i \alpha \Delta X}\right)}{\Delta X}+V \frac{\xi(\tau)\left(e^{i \beta \Delta Y}-1\right)}{\Delta Y}=M E_{c} \\
U \Psi(\tau)+\frac{1+N_{r}}{P_{r}} \frac{2 \xi(\tau)(\cos \beta \Delta Y-1)}{(\Delta Y)^{2}}+D_{u} \frac{2 \eta(\tau)(\cos \beta \Delta Y-1)}{(\Delta Y)^{2}}
\end{array}\right\}
$$

$$
\left.\begin{array}{l}
\frac{\eta^{\prime}(\tau)-\eta(\tau)}{\Delta \tau}+U \frac{\eta(\tau)\left(1-e^{-i \alpha \Delta X}\right)}{\Delta X}+V \frac{\eta(\tau)\left(e^{i \beta \Delta Y}-1\right)}{\Delta Y}=\frac{1}{S_{c}} \\
\frac{2 \eta(\tau)(\cos \beta \Delta Y-1)}{(\Delta Y)^{2}}+S_{r} \frac{2 \xi(\tau)(\cos \beta \Delta Y-1)}{(\Delta Y)^{2}}
\end{array}\right\}
$$

Now, the above system can be reduced as: 


$$
\begin{gathered}
\Psi^{\prime}(\tau)=A_{1} \Psi(\tau)+A_{2} \xi^{\prime}(\tau)+A_{3} \eta^{\prime}(\tau) \\
\xi^{\prime}(\tau)=A_{4} \xi(\tau)+A_{5} \Psi(\tau)+A_{6} \eta(\tau) \\
\eta^{\prime}(\tau)=A_{7} \eta(\tau)+A_{8} \xi(\tau)
\end{gathered}
$$

where,

$$
\begin{aligned}
& A_{1}=1-U \frac{\Delta \tau}{\Delta X}\left(1-e^{-i \alpha \Delta X}\right)-V \frac{\Delta \tau}{\Delta Y}\left(e^{i \beta \Delta Y}-1\right)-M \Delta \tau \\
& +\frac{2 \Delta \tau}{(\Delta Y)^{2}}(\cos \beta \Delta Y-1) \\
& A_{2}=G_{r} \Delta \tau \cos \alpha \\
& A_{3}=G_{m} \Delta \tau \cos \alpha \\
& A_{4}=1-U \frac{\Delta \tau}{\Delta X}\left(1-e^{-i \alpha \Delta X}\right)-V \frac{\Delta \tau}{\Delta Y}\left(e^{i \beta \Delta Y}-1\right)+ \\
& \frac{1+N_{r}}{P_{r}} \frac{2 \Delta \tau}{(\Delta Y)^{2}}(\cos \beta \Delta Y-1) \\
& A_{5}=M E_{c} U \Delta \tau \\
& A_{6}=\frac{2 D_{u} \Delta \tau}{(\Delta Y)^{2}}(\cos \beta \Delta Y-1) \\
& A_{7}=1-U \frac{\Delta \tau}{\Delta X}\left(1-e^{-i \alpha \Delta X}\right)-V \frac{\Delta \tau}{\Delta Y}\left(e^{i \beta \Delta Y}-1\right)+ \\
& \frac{2 \Delta \tau}{S_{c}(\Delta Y)^{2}}(\cos \beta \Delta Y-1) \\
& A_{8}=\frac{2 S_{r} \Delta \tau}{(\Delta Y)^{2}}(\cos \beta \Delta Y-1)
\end{aligned}
$$

Putting Eqns. (17) and (18) in Eq. (16) the following equation has been obtained as:

$$
\Psi^{\prime}(\tau)=A_{9} \Psi(\tau)+A_{10} \xi(\tau)+A_{11} \eta(\tau)
$$

where, $A_{9}=A_{1}+A_{2} A_{5}, A_{10}=A_{2} A_{4}+A_{3} A_{8}$, and $A_{11}=$ $A_{2} A_{6}+A_{3} A_{7}$.

The Eqns. (19), (17) and (18) become:

$$
\begin{gathered}
\Psi^{\prime}(\tau)=A_{9} \Psi(\tau)+A_{10} \xi(\tau)+A_{11} \eta(\tau) \\
\xi^{\prime}(\tau)=A_{4} \xi(\tau)+A_{5} \Psi(\tau)+A_{6} \eta(\tau) \\
\eta^{\prime}(\tau)=A_{7} \eta(\tau)+A_{8} \xi(\tau)
\end{gathered}
$$

And these equations are expressed in matrix notation as:

$$
\left[\begin{array}{l}
\Psi^{\prime} \\
\xi^{\prime} \\
\eta^{\prime}
\end{array}\right]=\left[\begin{array}{ccc}
A_{9} & A_{10} & A_{11} \\
A_{5} & A_{4} & A_{6} \\
0 & A_{8} & A_{7}
\end{array}\right]\left[\begin{array}{l}
\Psi \\
\xi \\
\eta
\end{array}\right]
$$

That is, $\omega^{\prime}=T \omega$.

where,

$$
\omega^{\prime}=\left[\begin{array}{c}
\Psi^{\prime} \\
\xi^{\prime} \\
\eta^{\prime}
\end{array}\right] ; T=\left[\begin{array}{ccc}
A_{9} & A_{10} & A_{11} \\
A_{5} & A_{4} & A_{6} \\
0 & A_{8} & A_{7}
\end{array}\right] ; \omega=\left[\begin{array}{c}
\Psi \\
\xi \\
\eta
\end{array}\right]
$$

Now we determine the eigenvalues of $T$, but this is very hard since $T$ is a third-order matrix. That is why we assume that $E_{c}$ and $D_{u}$ are very small which means tends to be zero. According to this condition $A_{9}=A_{1}, A_{5}=A_{6}=0$ and $A_{11}=$ $A_{3} A_{7}$. So,

$$
T=\left[\begin{array}{ccc}
A_{1} & A_{10} & A_{11} \\
0 & A_{4} & 0 \\
0 & A_{8} & A_{7}
\end{array}\right]
$$

From this above matrix, we get $\lambda_{1}=A_{1}, \lambda_{2}=A_{4}, \lambda_{3}=A_{7}$. For stability, $\lambda_{1}, \lambda_{2}$ and $\lambda_{3}$ are less than or equal to one in modulus. So the required condition is $\left|A_{1}\right| \leq 1,\left|A_{4}\right| \leq 1$ and $\left|A_{7}\right| \leq 1$.

Case-I: $\left|A_{1}\right| \leq 1$,

$$
\begin{aligned}
& A_{1}=(1-a-b-2 c-M \Delta \tau)+a(\cos \alpha \Delta X-i \sin \alpha \Delta X) \\
& +b(\cos \beta \Delta Y+i \sin \beta \Delta Y)+2 c \cos \beta \Delta Y
\end{aligned}
$$

Let $a=U \frac{\Delta \tau}{\Delta X}, b=|-V| \frac{\Delta \tau}{\Delta Y}$ and $c=\frac{\Delta \tau}{(\Delta Y)^{2}}$, then $A_{1}=(1-$ $a-b-2 c-M \Delta \tau)-a-b-2 c=1-[2(a+b+2 c)+$ $M \Delta \tau]$.

We get the maximum modulus of $A_{1}$ as $\alpha \Delta X=m \pi, \beta \Delta Y=n \pi$, ( $m \& n$ are integers and $A_{1}$ is real).

When $m \& n$ are odd integers:

$$
\begin{aligned}
& A_{1}=(1-a-b-2 c-M \Delta \tau)-a-b-2 c=1- \\
& {[2(a+b+2 c)+M \Delta \tau]}
\end{aligned}
$$

When $m \& n$ are even integers:

$$
A_{1}=(1-a-b-2 c-M \Delta \tau)+a+b+2 c=1-M \Delta \tau
$$

The condition requires that $\left|A_{1}\right| \leq 1$. For large $\Delta \tau$, the value of $A_{1}$ is relatively larger when $m \& n$ both are odd integers. So, $A_{1}=1-[2(a+b+2 c)+M \Delta \tau]$. To satisfy $\left|A_{1}\right| \leq 1$, where the least value is $A_{1}=-1$.

Thus, the condition is

$$
U \frac{\Delta \tau}{\Delta X}+|V| \frac{\Delta \tau}{\Delta Y}+2 \frac{\Delta \tau}{(\Delta Y)^{2}}+\frac{M \Delta \tau}{2} \leq 1
$$

Likewise, Case-II $\left(\left|A_{4}\right| \leq 1\right)$ gives,

$$
U \frac{\Delta \tau}{\Delta X}+|V| \frac{\Delta \tau}{\Delta Y}+\frac{1+N_{r}}{P_{r}} \frac{2 \Delta \tau}{(\Delta Y)^{2}} \leq 1
$$

Also, Case-III $\left(\left|A_{7}\right| \leq 1\right)$ gives

$$
U \frac{\Delta \tau}{\Delta X}+|V| \frac{\Delta \tau}{\Delta Y}+\frac{2}{S_{c}} \frac{\Delta \tau}{(\Delta Y)^{2}} \leq 1
$$

Since from initial condition $U=V=0$ at $\tau=0$, the Eqns. (20), (21), (22) give $M \leq 336, P_{r} \geq 0.24$ for $N_{r}=0.5$, and $N_{r} \leq$ 3.44 for $P_{r}=0.71, S_{c} \geq 0.16$ respectively.

Hence the convergence criteria are $M \leq 336, P_{r} \geq 0.24$ for $N_{r}=0.5, N_{r} \leq 3.44$ for $P_{r}=0.71$, and $S_{c} \geq 0.16$. 


\section{NUMERICAL SOLUTIONS}

Same number of finite difference equations have been determined from the previous non-dimensional equations. For this purpose, a rectangular region is chosen which has two axes $\left(X\right.$ and $Y$ ). Let $X_{\max }(=100)$ i.e. $X$ ranges from 0 to 100 and $Y_{\max }(=25)$ as corresponding to $Y \rightarrow \infty$. Consider, $m=100$ and $n=100$ grids in the $X$ and $Y$-directions respectively as visualised in Figure 2. The flow zone has been discretized in backward difference for the terms in the first-order derivative along the $X$ axis and forward difference along the $Y$ axis while the central difference does the tasking of higher-order discretizations. The time value of a particular point is calculated by importing the time value of the preceding point. The mesh sizes of $X$ and $Y$ are $\Delta X, \Delta Y$ where $\Delta X=1.0(0 \leq x \leq 100)$; $\Delta Y=0.25(0 \leq y \leq 25)$ with $\Delta \tau=0.005$.

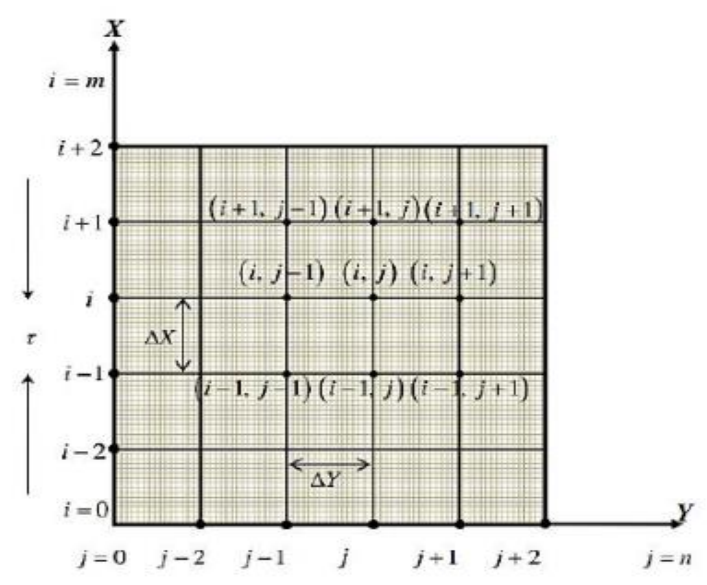

Figure 2. Grid sheet

$i$ and $j$ refer the specific grid points with $X$ and $Y$ coordinates respectively while the time value, $\tau=n \Delta \tau$, where $\mathrm{n}=0$, $1,2,3,4 \ldots$

\section{RESULTS AND DISCUSSION}

The upshots of the two-dimensional transient flow have been understood and revealed in terms of sundry dimensionless parameters by solving the governing system of PDEs in an efficient EFDM procedure. The results have been determined for $\tau=40, m=100$ and $n=100$. The results are restricted to $\mathrm{P}_{\mathrm{r}}=0.30\left(\mathrm{P}_{\mathrm{r}}\right.$ around $0.16-0.70$ for gaseous mixtures), $P_{r}=0.63$ ( $P_{r}$ for Oxygen), $P_{r}=0.71\left(P_{r}\right.$ for air at $\left.20^{\circ} \mathrm{C}\right), \mathrm{P}_{\mathrm{r}}=1.0\left(\mathrm{P}_{\mathrm{r}}\right.$ for saltwater at $\left.20^{\circ} \mathrm{C}\right)$, and $\mathrm{P}_{\mathrm{r}}=1.38\left(\mathrm{P}_{\mathrm{r}}\right.$ for gaseous Ammonia). According to the convergence conditions the values of Schmidt number $\left(\mathrm{S}_{\mathrm{c}}\right)$ are taken 0.22 (for Hydrogen), 0.30 (for Helium), 0.60 (for water vapor), 0.78 (for Ammonia) and 1.0 (for Methanol at $25^{\circ} \mathrm{C}$ and $1 \mathrm{~atm}$ ). However, the arbitrary choices have been taken for the rest of the parameters accordingly with the converging criteria. The profiles of velocity, temperature, and concentration with the influence of various parameters have been illustrated pictorially in Figures 3-32.

The velocity, temperature, and concentration profiles against magnetic parameter $M$ have been illustrated in Figures 3-5. In Figures 3-4, fluid velocity and temperature show the decreasing (for $Y \leq 18$ approximately) and increasing (for $Y \leq 10$ approximately) trends respectively near the porous wall with the rising values of $M$ and thereafter start to fall. But fluid concentration seems to be increase with the rise of $M$ as shown in Figure 5.

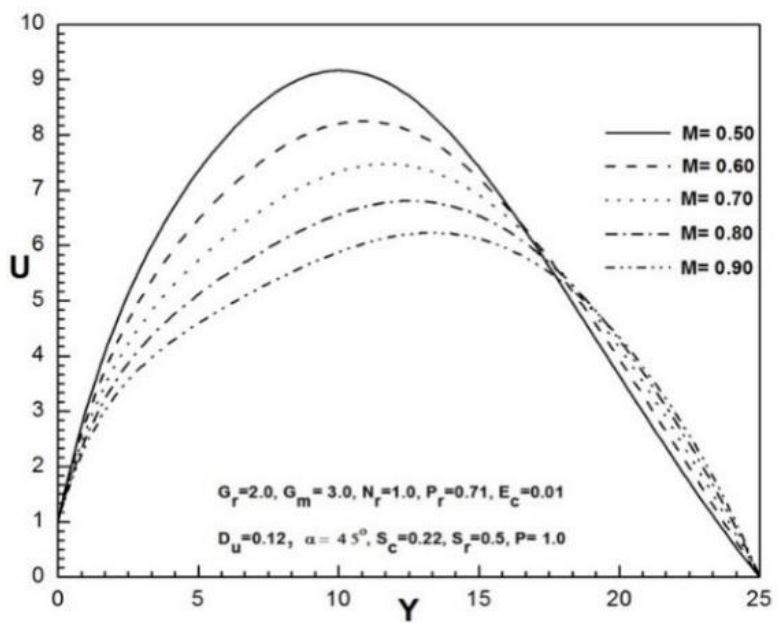

Figure 3. Velocity profiles for $M$

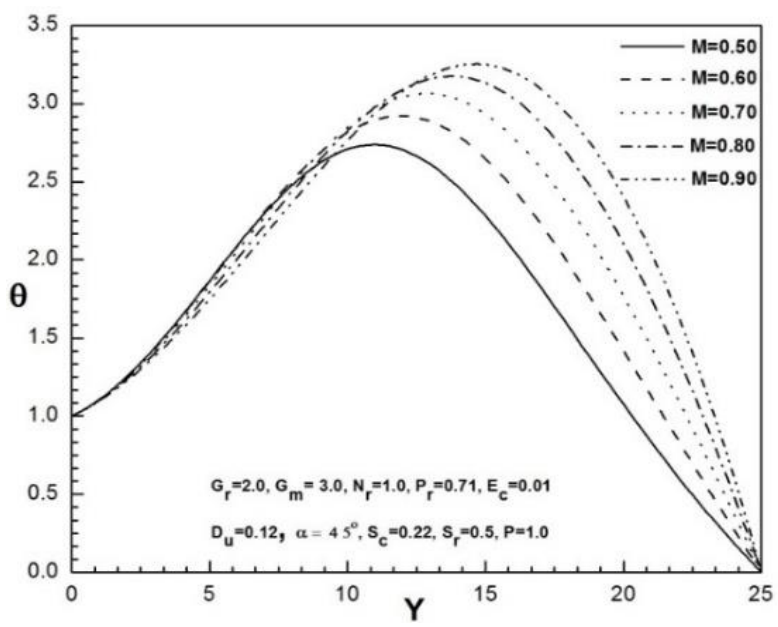

Figure 4. Temperature profiles for $M$

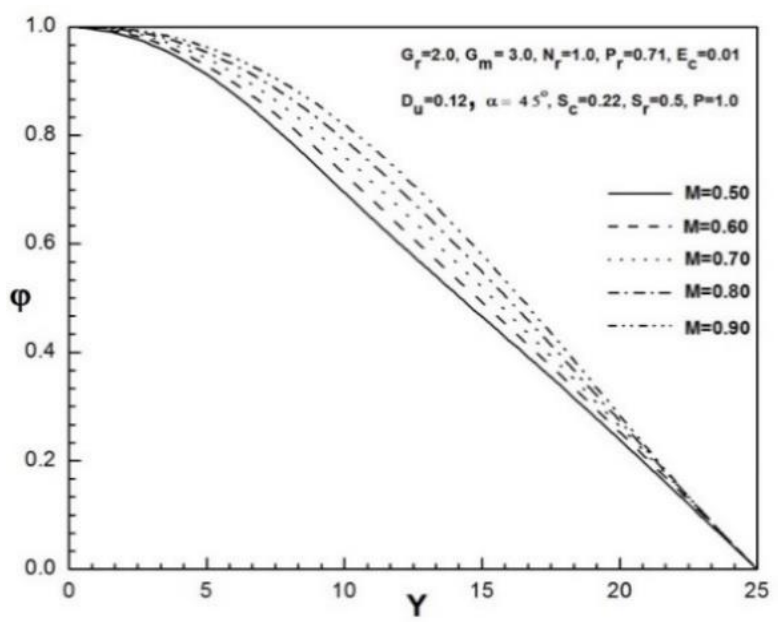

Figure 5. Concentration profiles for $M$

The features of Grashof number of heat transfer $G_{r}$ on flow profiles have been depicted in Figures 6-8. In Figures 6-7, flow velocity (for $Y \leq 15$ approximately) and temperature (for $Y \leq 14$ approximately) both demonstrate the rising trends contiguous to the plate with the intensification of the values of $G_{r}$ and thereafter for the rest of the boundary layer zone, those begin 
to suppress. In Figure 8, it is obvious that the boundary layer for concentration contracts in the flow tract for rising estimation of $G_{r}$.

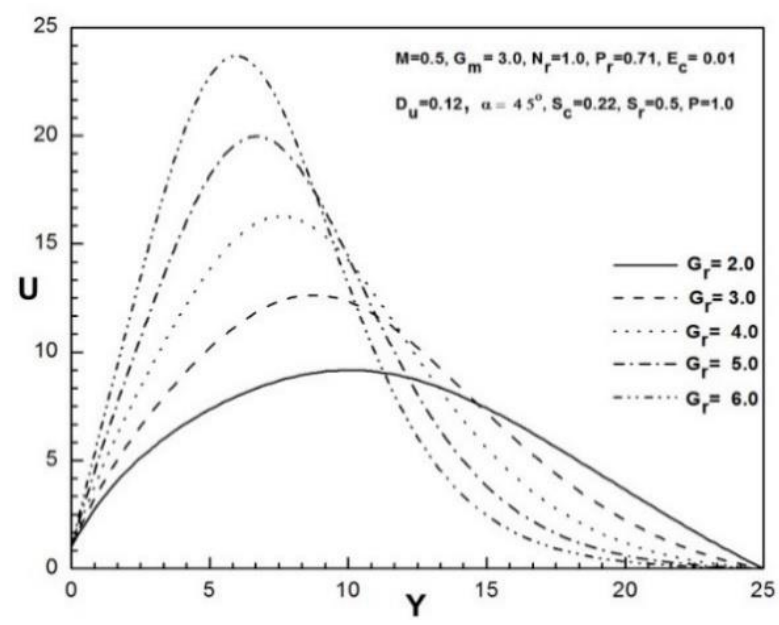

Figure 6. Velocity profiles for $G_{r}$

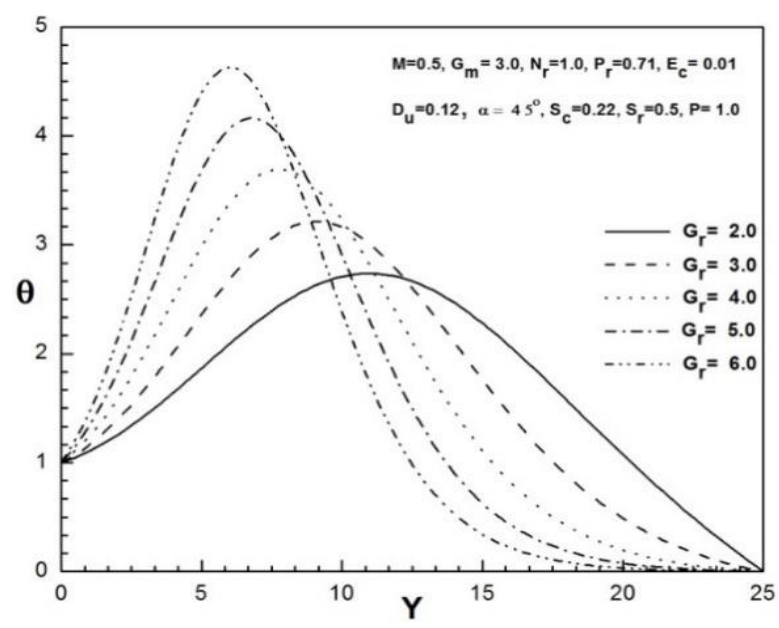

Figure 7. Temperature profiles for $G_{r}$

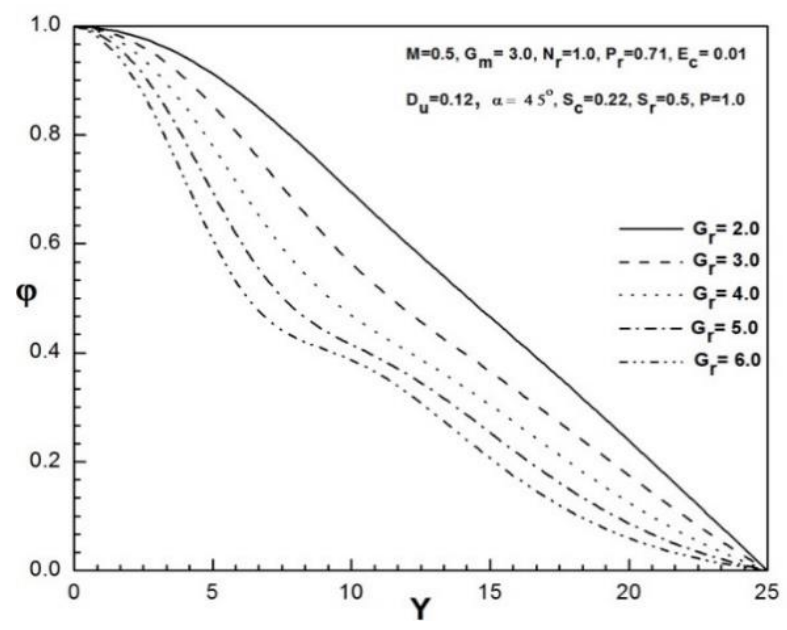

Figure 8. Concentration profiles for $G_{r}$

In Figures 9-11, the impacts of Grashof number of mass transfer $G_{m}$ on flow profiles have been depicted. Concentration level is marked to diminish with the increase of $G_{m}$, while the flow velocity (for $Y \leq 14$ approximately) and temperature (for $Y \leq 13$ approximately) both rise and then for the rest of the boundary layer tract, they fall down for the rising estimations of $G_{m}$.

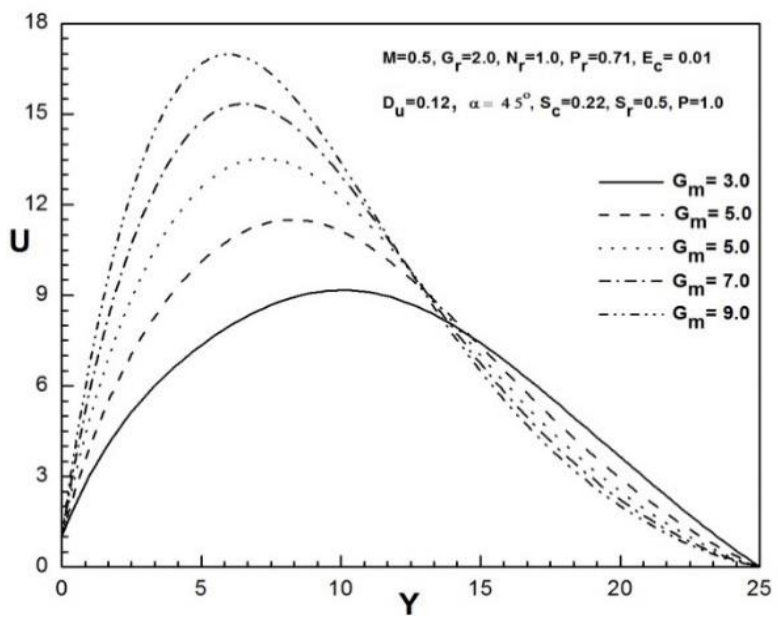

Figure 9. Velocity profiles for $G_{m}$

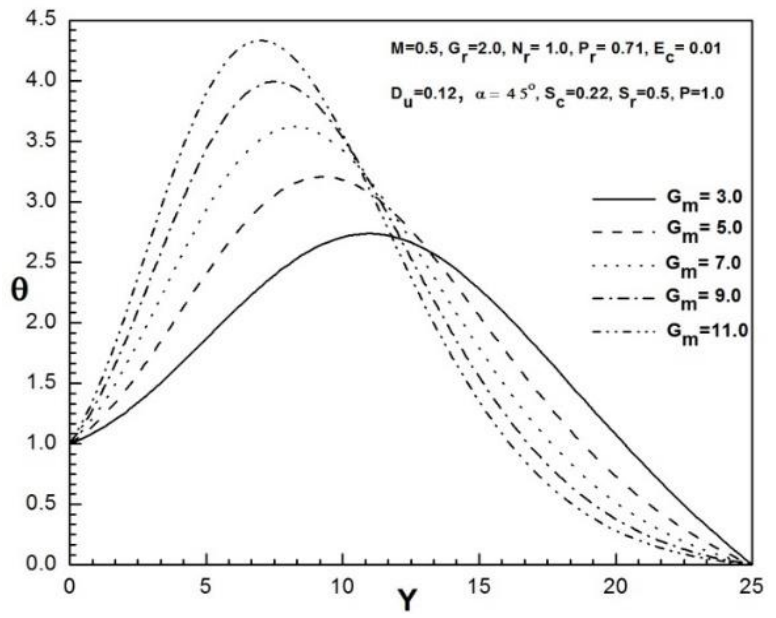

Figure 10. Temperature profiles for $G_{m}$

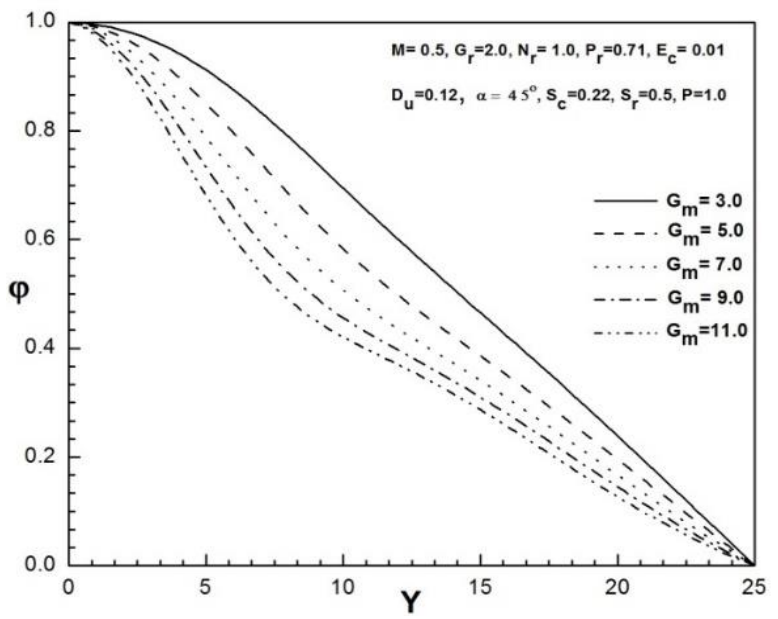

Figure 11. Concentration profiles for $G_{m}$

The graphical representations of thermal radiation parameter $N_{r}$ on the velocity of the fluid along with the temperature and concentration distributions have been demonstrated in Figures 12-14. It is observed from Figures 1213 that both the velocity and temperature first decrease until $Y \leq 17$ (approximately) and $Y \leq 18$ (approximately) respectively, 
and then develops with the increase of $N_{r}$. A minor increasing effect has been noticed in concentration from Figure 14 with the increase of $N_{r}$.

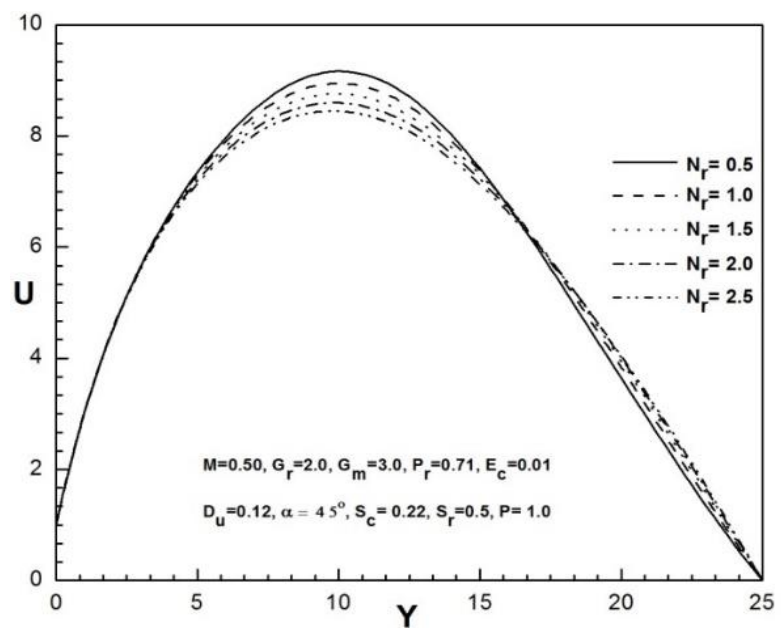

Figure 12. Velocity profiles for $N_{r}$

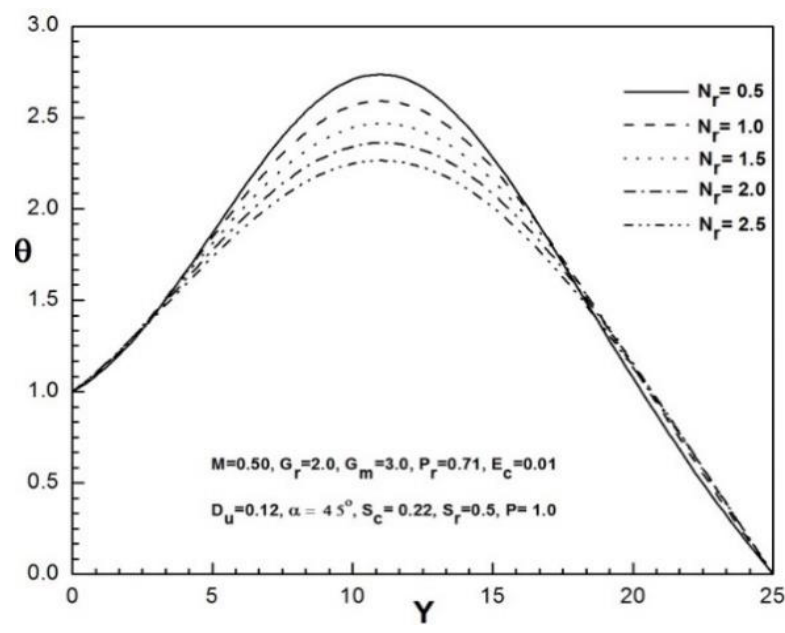

Figure 13. Temperature profiles for $N_{r}$

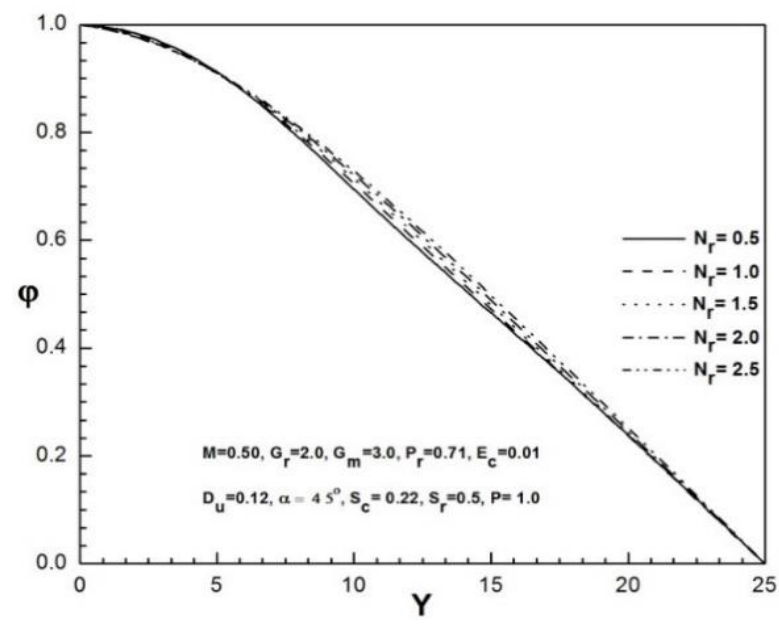

Figure 14. Concentration profiles for $N_{r}$

The behavior of the Prandtl number $P_{r}$ on the velocity field, fluid temperature, and fluid concentration has been illustrated in Figures 15-17. In Figures 15-16, fluid velocity and temperature both show first increasing impact until $Y \leq 16$ (approximately) and $Y \leq 19$ (approximately) respectively and then reveal decreasing impact with the rise of $P_{r}$. In Figure 17, fluid concentration discloses marginal decrement with the increase of $P_{r}$.

In Figures 18-20, the behavior of Eckert number $E_{c}$ on fluid velocity, temperature, and concentration has been shown. The concentration boundary layer is found to decrease with the rise of $E_{c}$ from Figure 20, while the fluid velocity (for $Y \leq 16$ approximately) and temperature (for $Y \leq 17$ approximately) both increase and then decrease for the increasing values $E_{c}$.

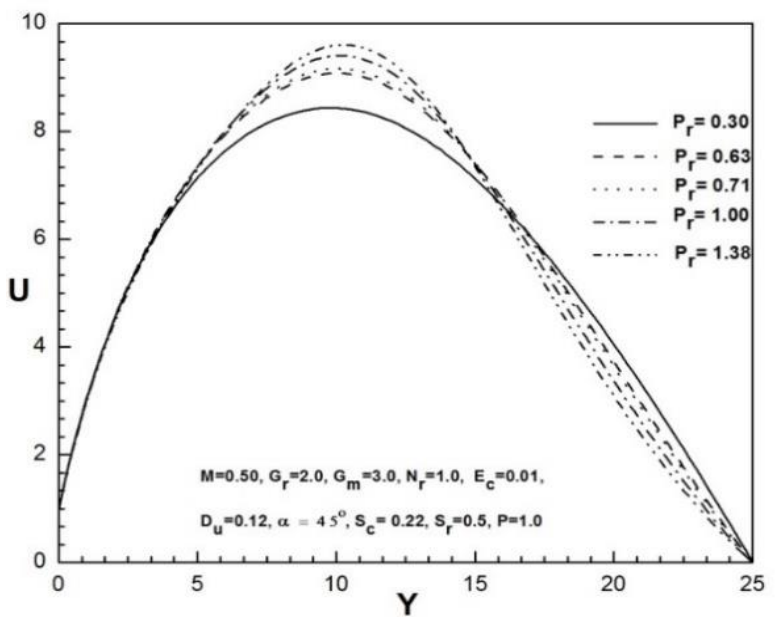

Figure 15. Velocity profiles for $P_{r}$

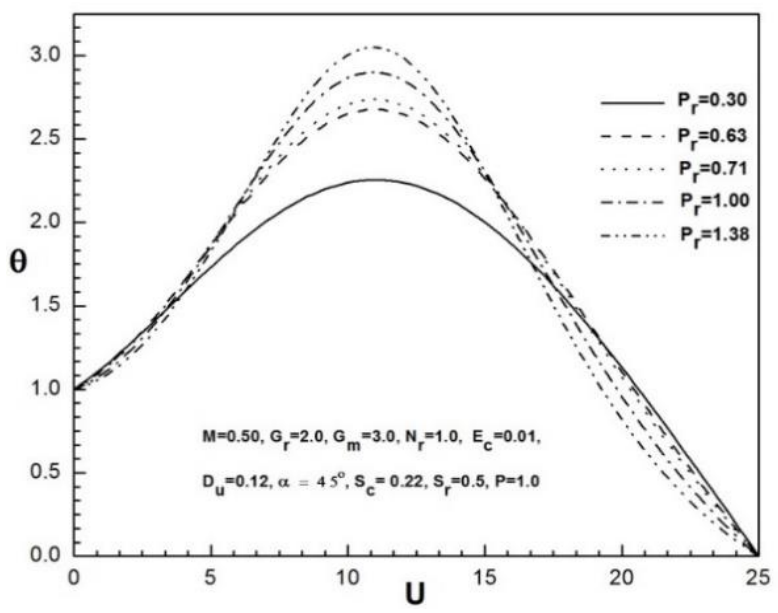

Figure 16. Temperature profiles for $P_{r}$

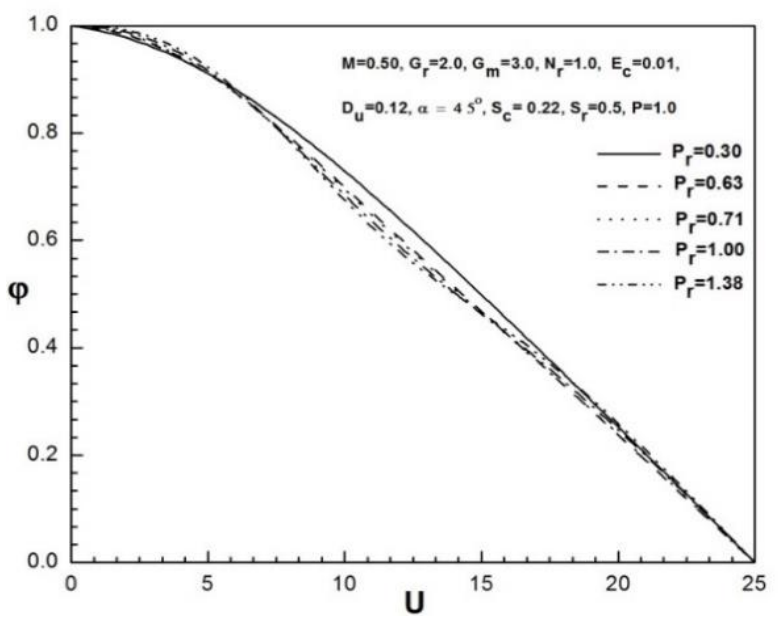

Figure 17. Concentration profiles for $P_{r}$ 


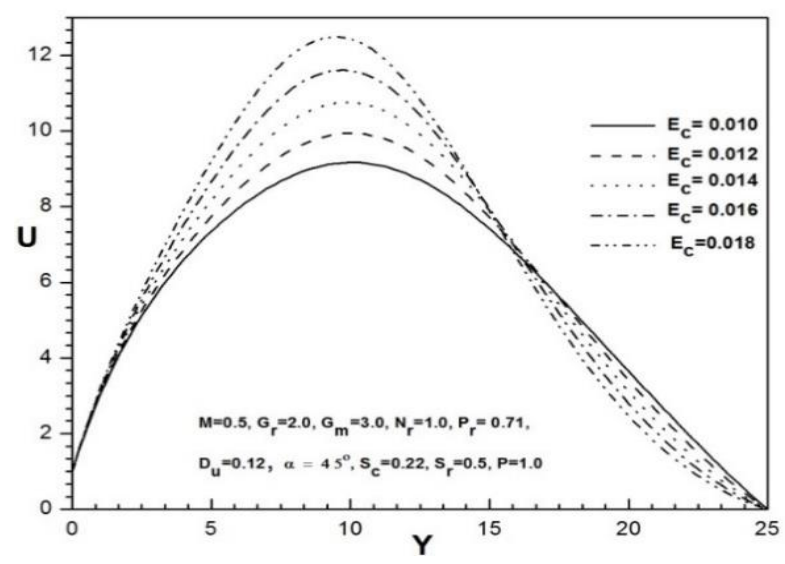

Figure 18. Velocity profiles for $E_{c}$

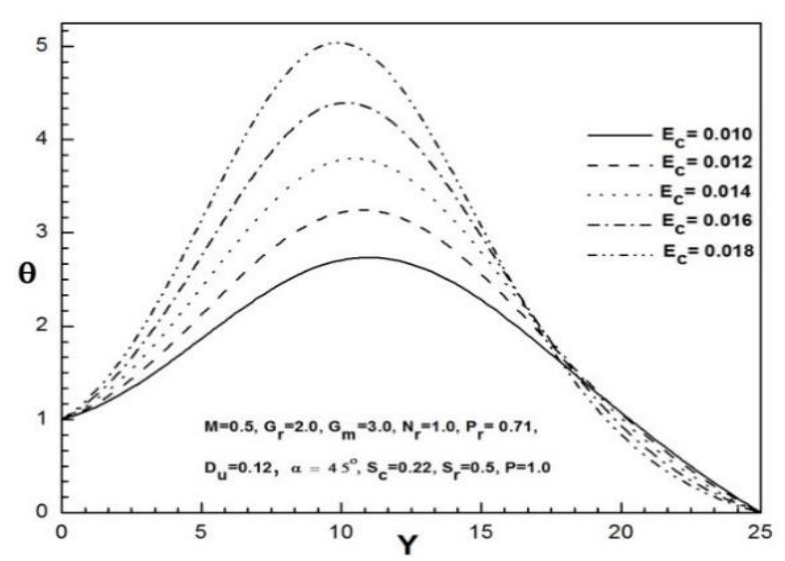

Figure 19. Temperature profiles for $E_{c}$

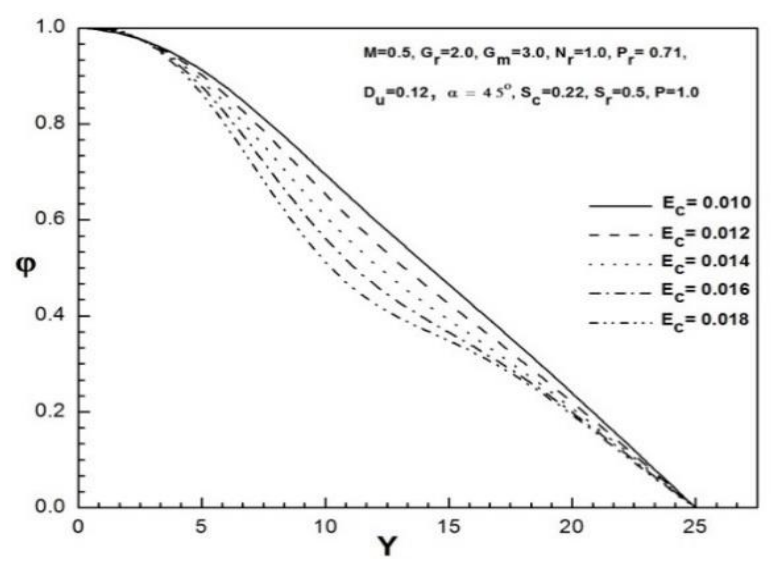

Figure 20. Concentration profiles for $E_{c}$

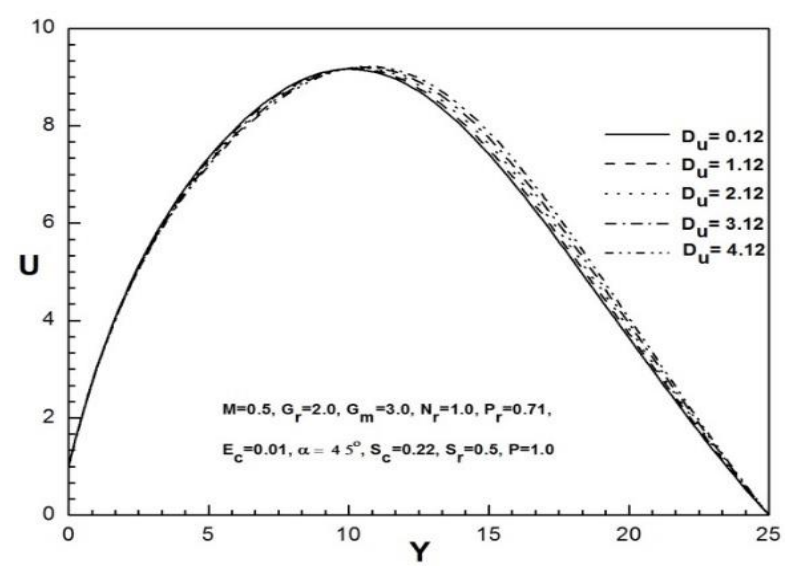

Figure 21. Velocity profiles for $D_{u}$

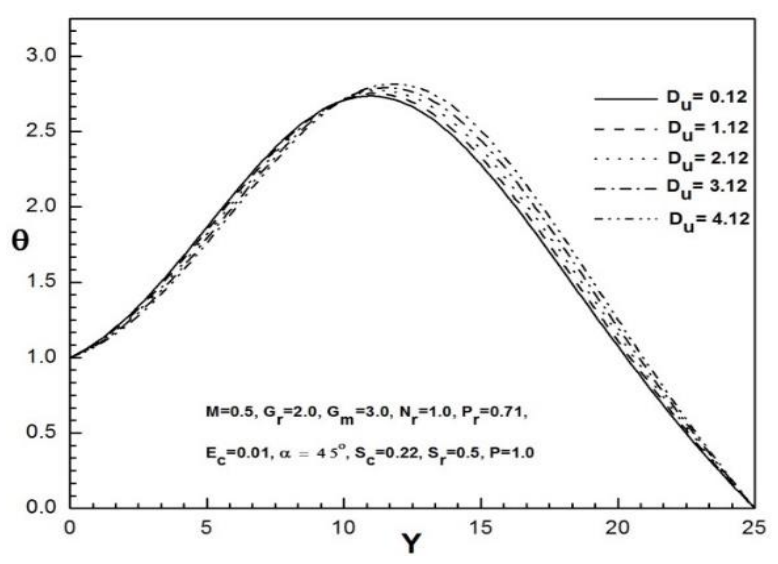

Figure 22. Temperature profiles for $D_{u}$

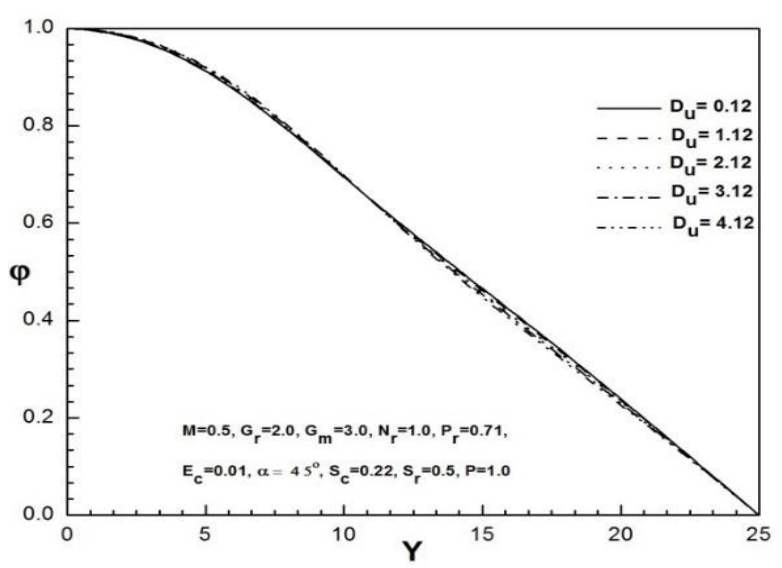

Figure 23. Concentration profiles for $D_{u}$

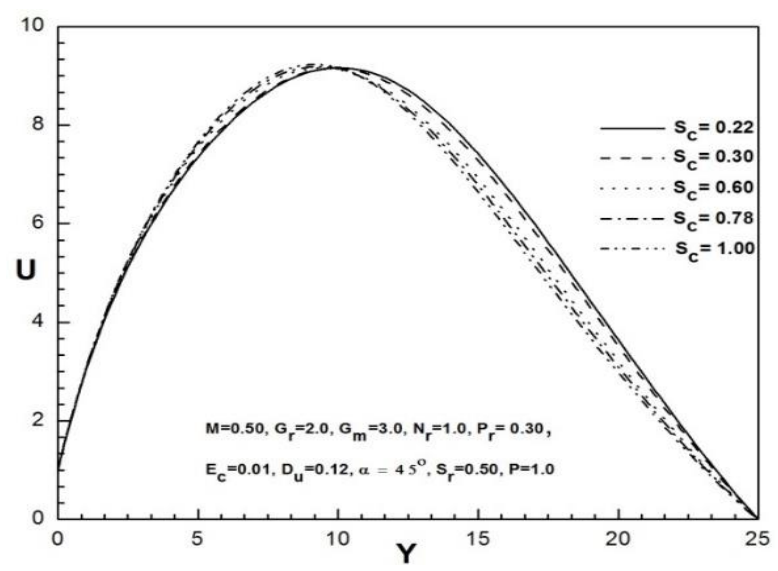

Figure 24. Velocity profiles for $S_{c}$

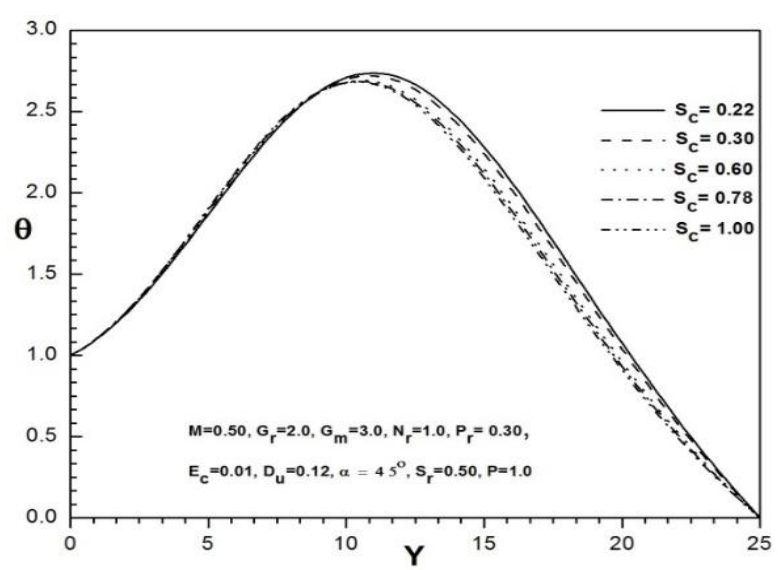

Figure 25. Temperature profiles for $S_{c}$ 


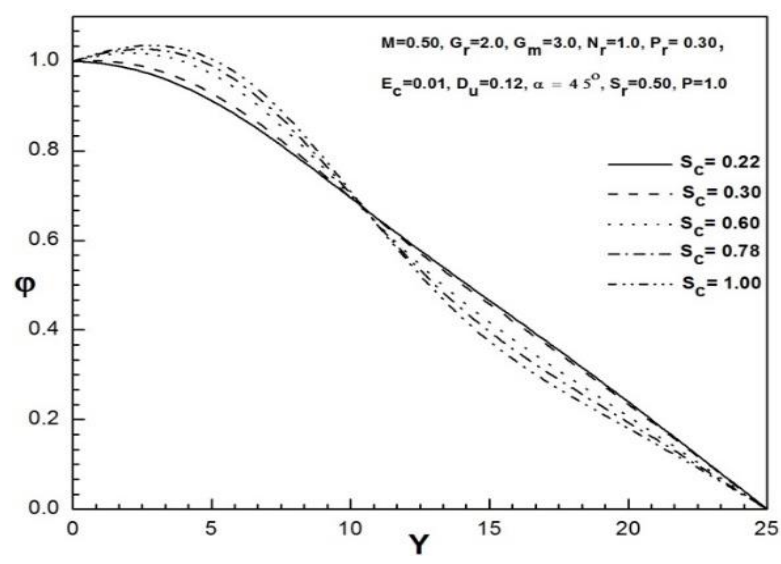

Figure 26. Concentration profiles for $S_{c}$

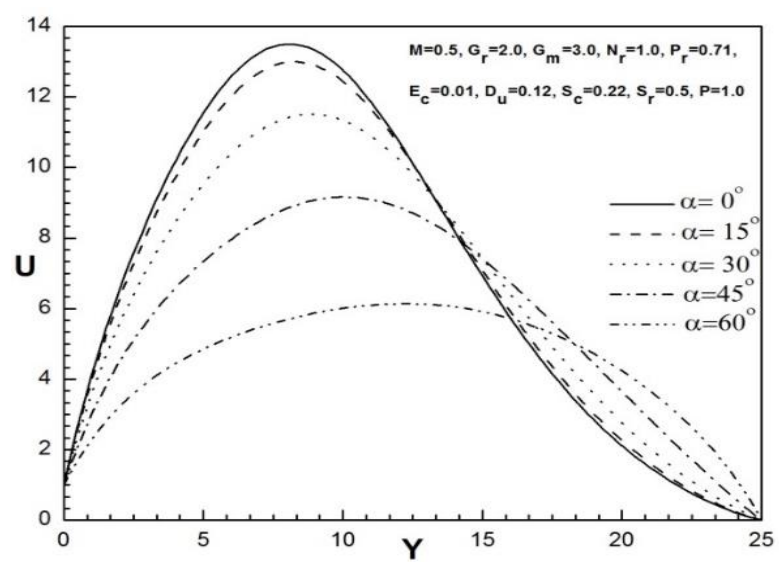

Figure 27. Velocity profiles for $\alpha$

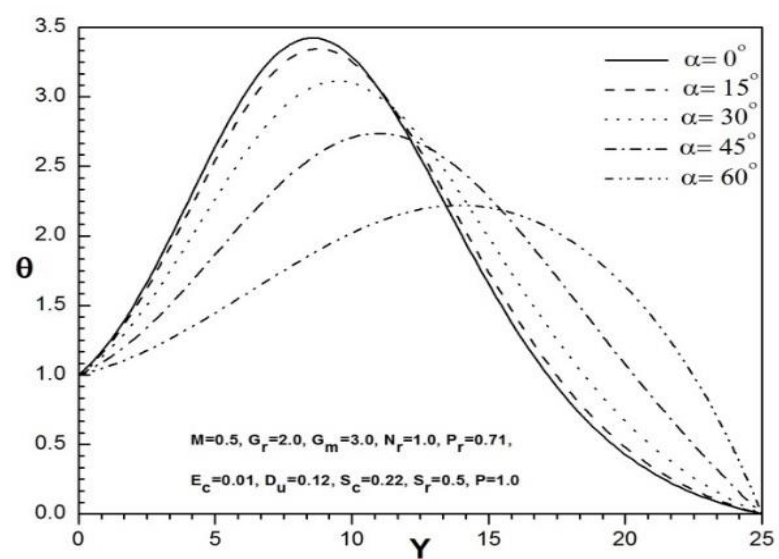

Figure 28. Temperature profiles for $\alpha$

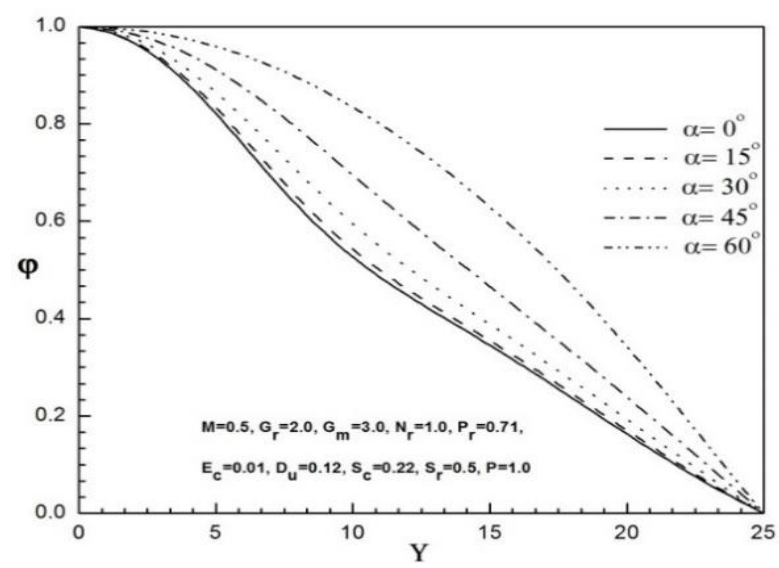

Figure 29. Concentration profiles for $\alpha$

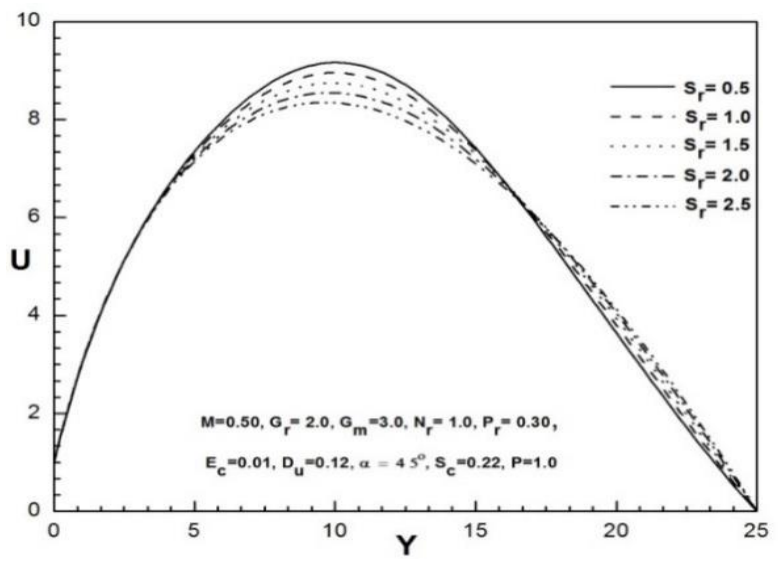

Figure 30. Velocity profiles for $S_{r}$

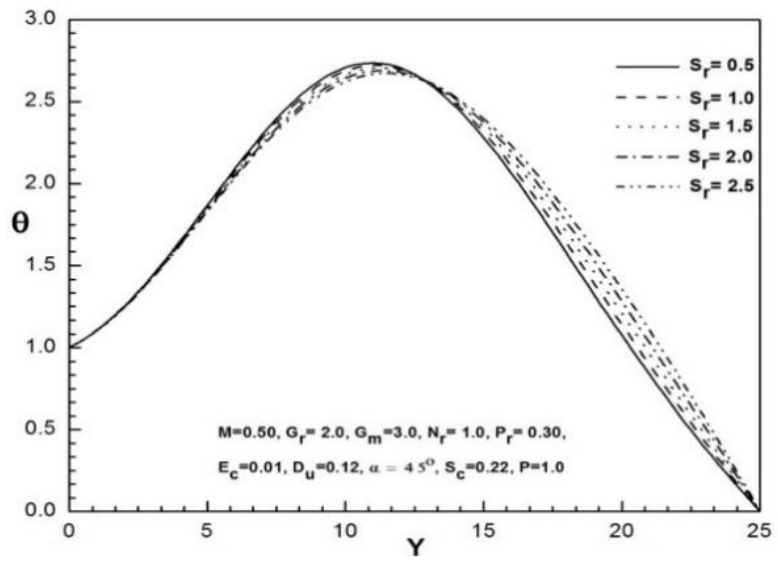

Figure 31. Temperature profiles for $S_{r}$

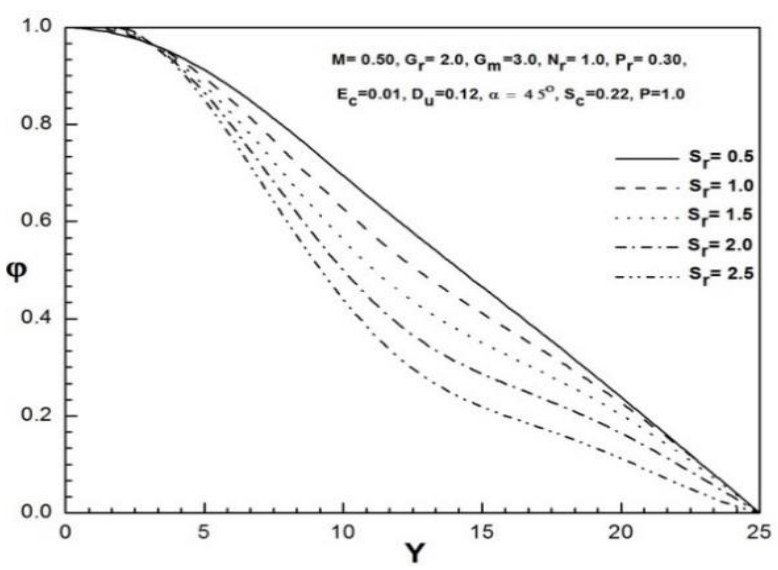

Figure 32. Concentration profiles for $S_{r}$

The velocity, temperature, and concentration profiles for different estimations of the Dufour number $D_{u}$ have been illustrated in Figures 21-23. In Figures 21-22, fluid velocity discloses a gradual increasing change, while temperature profile marginally decreases near the plate and then raises with the raise of $D_{u}$. The negligible changing behavior of fluid concentration has been counted in Figure 23 with the increase of $D_{u}$.

The graphical representation of Schmidt number $S_{c}$ on fluid velocity, temperature, and concentration have been depicted in Figures 24-26. From Figures 24-25, it is noted that the flow velocity decreases until $Y \leq 11$ (approximately) and then increases, while the temperature profile falls with the 
enhancement of $S_{c}$. The concentration near the wall hikes up and then after $Y \leq 10$ (approximately), it starts to decrease with the raise of $S_{c}$.

The influences of different inclined angles $\alpha$ on the flow velocity, temperature, and concentration are illustrated in Figures 27-29. Figures 27 and 28 disclose that both the flow velocity and temperature substantially suppress contiguous to the plate and then increasingly project through the rest of the boundary layer regime with the raise of $\alpha$. The fluid concentration in Figure 29 exhibits that it enhances with the raise of $\alpha$.

The velocity, temperature, and concentration have been illustrated in Figures 30-32 against Soret number $S_{r}$. In those figures, decrement in fluid concentration has been marked with the larger values of $S_{r}$. The velocity and the temperature both first fall down and then climb up with the increase of $S_{r}$.

\section{CONCLUSION}

The EFDM numerical reckoning on transient MHD radiative flow over a porous plate of an inclination with Soret and Dufour impacts has been presented in the current study. The salient upshots of flow phenomena are mentioned and discussed for different important parameters. The concluding remarks for the present numerical investigation are specified below:

1. Fluid velocity boosts up near the wall with the enhancement of $G_{r}, G_{m}, P_{r}, E_{c}$ and diminish for the increasing values of $M, N_{r}, \alpha$.

2. The fluid temperature has been reported to develop with the increase of $P_{r}$, and $E_{c}$ fall with the increase of $N_{r}$, and $S_{c}$, vicinity to the plate.

3. Fluid concentration has been marked to dense with the raise of $M$, and $\alpha$ and fall with the rise of $G_{r}, G_{m}, E_{c}$, and $S_{r}$.

4. A minor effect has been seen in fluid velocity for $D_{u}$ and in fluid concentration for $N_{r}, D_{u}$.

In general, it is identified that the velocity tends to increase or decrease consecutively towards the plate and then decrease or increase slowly off the plate. The same behavior has been seen for fluid temperature. Some parameters such as $N_{r}, D_{u}$ have minor effects on concentration.

\section{REFERENCES}

[1] Alfvén, H. (1942). Existence of electromagnetichydrodynamic waves. Nature, 150(3805): 405-406. https://doi.org/10.1038/150405d0

[2] Sheri, S.R., Modugula, P. (2016). Heat and mass transfer effects on unsteady MHD flow over an inclined porous plate embedded in porous medium with Soret-Dufour and chemical reaction. International Journal of Applied and Computational Mathematics, 3(2): 1289-1306. https://doi.org/10.1007/s40819-016-0177-4

[3] Makinde, O.D., Khan, Z.H., Ahmad, R., Haq, R.U., Khan, W.A. (2019). Unsteady MHD flow in a porous channel with thermal radiation and heat source/sink. International Journal of Applied and Computational Mathematics, 5: 59. https://doi.org/10.1007/s40819-019-0644-9

[4] Subhas Abel, M., Begum, G. (2008). Heat transfer in MHD viscoelastic fluid flow on stretching sheet with heat source/sink, viscous dissipation, stress work, and radiation for the case of large Prandtl number. Chemical Engineering Communications, 195(12): 1503-1523. https://doi.org/10.1080/00986440801907300

[5] Chamkha, A.J., Mujtaba, M., Quadri, A., Issa, C. (2003). Thermal radiation effects on MHD forced convection flow adjacent to a non-isothermal wedge in the presence of a heat source or sink. Heat and Mass Transfer, 39(4): 305-312. https://doi.org/10.1007/s00231-002-0353-4

[6] Raptis, A. (2017). Effects of thermal radiation on the MHD flow past a vertical plate. Journal of Engineering Thermophysics, 26(1): 53-59. https://doi.org/10.1134/s1810232817010064

[7] Ahmed, F., Iqbal, M. (2020). Heat transfer analysis of MHD power law nano fluid flow through annular Sector Duct. Journal of Thermal Science, 29: 169-181. https://doi.org/10.1007/s11630-019-1126-4

[8] Kiyasatfar, M., Pourmahmoud, N. (2016). Laminar MHD flow and heat transfer of power-law fluids in square microchannels. International Journal of Thermal Sciences, 99: 26-35. https://doi.org/10.1016/j.ijthermalsci.2015.07.031

[9] Ravikumar, V., Raju, M.C., Raju, G.S.S. (2012). MHD three dimensional Couette flow past a porous plate with heat transfer. IOSR Journal of Mathematics (IOSRJM), 1(3): 3-9. https://doi.org/10.9790/5728-0130309

[10] Raju, M.C., Varma, S.V.K., Reddy, N.A. (2012). Radiation and mass transfer effects on a free convection flow through a porous medium bounded by a vertical surface. Journal of Future Engineering and Technology, 7(2): 7-12. http://dx.doi.org/10.26634/jfet.7.2.1757

[11] Reddy, T.S., Raju, M.C., Varma, S.V.K. (2013). Unsteady MHD radiative and chemically reactive free convection flow near a moving vertical plate in porous medium. Journal of Applied Fluid Mechanics, 6(3): 443451. https://doi.org/10.36884/jafm.6.03.21306

[12] Reddy, P.G., Umamaheswar, M., Raju, M.C., Varma, S.V.K. (2016). Magneto-convective and radiation absorption fluid flow past an exponentially accelerated vertical porous plate with variable temperature and concentration. International Journal of Mathematics Trends and Technology (IJMTT), 31(1): 26-33. https://doi.org/10.14445/22315373/IJMTT-V31P507

[13] Izadi, M., Shahivand, I., Mehryan, S.A., Hasan, M.S., Lorenzini, G. (2020). Magneto-hydrodynamic flow of micropolar nanofluid containing motile microorganisms passing over a vertical stretching sheet with magnetic field dependent viscosity. Journal of Engineering Thermophysics, 29(4): 632-656. https://doi.org/10.1134/S1810232820040116

[14] Islam, M.M., Mollah, M.T., Hasan, M.S., Alam, M.M. (2017). Numerical solution of unsteady viscous compressible fluid flow along a porous plate with induced magnetic field. Modelling, Measurement and Control B, 86(4): 850-863. https://doi.org/10.18280/mmc_b.860403

[15] Mollah, M.T., Islam, M.M., Khatun, S., Alam, M.M. (2019). MHD generalized Couette flow and heat transfer on Bingham Fluid through porous parallel plates. Mathematical Modeling of Engineering Problems, 6(4): 483-490. https://doi.org/10.18280/mmep.060402

[16] Mollah, M.T. (2019). EMHD laminar flow of Bingham fluid between two parallel Riga plates. International Journal of Heat and Technology, 37(2): 641-648. https://doi.org/10.18280/ijht.370236 
[17] Reddy, V., Neelima, A., Thiagarajan, S. (2019). Finite difference solutions of MHD natural convective RivlinEricksen fluid flow past a vertically inclined porous plate in presence of thermal diffusion, diffusion thermo, heat and mass transfer effects. International Journal of Applied Engineering Research, 14(3): 703-716.

[18] Pandya, N., Shukla, A.K. (2014). Soret-Dufour and radiation effect on unsteady MHD flow over an inclined porous plate embedded in porous medium with viscous dissipation. International Journal of Advances in Applied Mathematics and Mechanics, 2(1): 107-119.

[19] Omokhuale, E. Altine, M.M., Yusuf, A. (2019). Unsteady heat and mass transfer magnetohydrodynamic (MHD) convective Couette flow with thermal radiation using finite element method (FEM). Caliphate Journal of Science \& Technology (CaJoST), 1: 61-70.

[20] Khan, M.I., Hayat, T., Khan, M.I., Alsaedi, A. (2017). A modified homogeneous-heterogeneous reactions for MHD stagnation flow with viscous dissipation and Joule heating. International Journal of Heat and Mass Transfer, 113: 310-317. https://doi.org/10.1016/j.ijheatmasstransfer

[21] Waqas, M., Farooq, M., Khan, M.I., Alsaedi, A., Hayat, T., Yasmeen, T. (2016). Magnetohydrodynamic (MHD) mixed convection flow of micropolar liquid due to nonlinear stretched sheet with convective condition. International Journal of Heat and Mass Transfer, 102: 766-772. https://doi.org/10.1016/j.ijheatmasstransfer

[22] Malga, B.S., Kishan, N. (2014). Finite element analysis for unsteady MHD heat and mass transfer free convection flow of polar fluids past a vertical moving porous plate in a porous medium with heat generation and thermal diffusion. Journal of Naval Architecture and Marine Engineering, 11(1). https://doi.org/10.3329/jname.v11i1.12844

[23] Makinde, O.D. (2011). Heat and mass transfer by MHD mixed convection stagnation point flow toward a vertical plate embedded in a highly porous medium with radiation and internal heat generation. Meccanica, 47(5): 1173-1184. https://doi.org/10.1007/s11012-011-9502-5

[24] Anand, V.W.J., Ganesh, S., Chandrasekar, P., Jayaraman, J., Mariadhas, A. (2021). Unsteady MHD Couette viscous fluid flow through silver metallic parallel plates with an inclined magnetic field and angular velocity subjected to constant suction at lower plate. Materials Today: $\quad$ Proceedings, 44: 3919-3923. https://doi.org/10.1016/j.matpr.2021.02.389

[25] Tassaddiq, A. (2019). MHD flow of a fractional second grade fluid over an inclined heated plate. Chaos, Solitons \& Fractals, 123: 341-346. https://doi.org/10.1016/j.chaos.2019.04.029

[26] Pattnaik, J.R., Dash, G.C., Singh, S. (2017). Radiation and mass transfer effects on MHD flow through porous medium past an exponentially accelerated inclined plate with variable temperature. Ain Shams Engineering Journal, $\quad 8(1)$ : $67-75$ https://doi.org/10.1016/j.asej.2015.08.014

[27] Sabu, A.S., Mathew, A., Neethu, T.S., Anil George, K. (2020). Statistical analysis of MHD convective ferronanofluid flow through an inclined channel with hall current, heat source and Soret effect. Thermal Science and Engineering Progress, 22: 100816. https://doi.org/10.1016/j.tsep.2020.100816
[28] Khashi'ie, N.S., Arifin, N.M., Pop, I. (2021). Magnetohydrodynamics (MHD) boundary layer flow of hybrid nanofluid over a moving plate with Joule heating, Alexandria Engineering Journal. https://doi.org/10.1016/j.aej.2021.07.032

[29] Poddar, S., Islam, M.M., Ferdouse, J., Alam, M.M. (2021). Characteristical analysis of MHD heat and mass transfer dissipative and radiating fluid flow with magnetic field induction and suction. SN Applied Sciences, 3: 470. https://doi.org/10.1007/s42452-02104452-4

[30] Ouaf, M.E.M. (2005). Exact solution of thermal radiation on MHD flow over a stretching porous sheet. Applied Mathematics and Computation, 170(2): 1117-1125. https://doi.org/10.1016/j.amc.2005.01.010

[31] Alam, M.S., Rahman, M.M., Sattar, M.A. (2008). Effects of variable suction and thermophoresis on steady MHD combined free-forced convective heat and mass transfer flow over a semi-infinite permeable inclined plate in the presence of thermal radiation. International Journal of Thermal Science, 47(6): 758-765. https://doi.org/10.1016/j.ijthermalsci.2007.06.006

[32] Pal, D., Talukdar, B. (2010). Buoyancy and chemical reaction effects on MHD mixed convection heat and mass transfer in a porous medium with thermal radiation and Ohmic heating. Communications in Nonlinear Science and Numerical Simulation, 15(10): 2878-2893. https://doi.org/10.1016/j.cnsns.2009.10.029

[33] Seth, G.S., Nandkeolyar, R., Ansari, M.S. (2013). Effects of thermal radiation and rotation on unsteady hydromagnetic free convection flow past an impulsively moving vertical plate with ramped temperature in a porous medium. Journal of Applied Fluid Mechanics, 6(1): 27-38. https://doi.org/10.36884/jafm.6.01.19478

[34] Seth, G.S., Kumbhakar, B., Sarkar, S. (2014). Unsteady hydromagnetic natural convection flow with heat and mass transfer of a thermally radiating and chemically reactive fluid past a vertical plate with Newtonian heating and time dependent free-stream. International Journal of Heat and Technology, 32(1-2): 87-94. https://doi.org/10.18280/ijht.320113

[35] Mollah, M.T., Poddar S., Islam, M.M., Alam, M.M. (2021). Non-isothermal Bingham fluid flow between two horizontal parallel plates with Ion-slip and Hall currents. SN Applied Sciences, 3: 115. https://doi.org/10.1007/s42452-020-04012-2

[36] Iva, L.M., Hasan, M.S., Paul, S.K., Mondal, R.N. (2018). MHD free convection heat and mass transfer flow over a vertical porous plate in a rotating system with Hall current, heat source and suction. International Journal of Advances in Applied Mathematics and Mechanics, 6(1): 49-64.

[37] Reza-E-Rabbi, S., Ahmmed, S.F., Arifuzzaman, S.M., Sarkar, T., Khan, M.S. (2019). Computational modeling of multiphase fluid flow behaviour over a stretching sheet in the presence of nanoparticles. Engineering Science and Technology, an International Journal, 23(3): 605-617. https://doi.org/10.1016/j.jestch.2019.07.006

[38] Khatun, S., Islam, M.M., Mollah, M.T., Poddar, S., Alam M.M. (2021). EMHD radiating fluid flow along a vertical Riga plate with suction in a rotating system. SN Applied Sciences, 3(452). https://doi.org/10.1007/s42452-02104444-4 